\title{
Vibration-Induced Friction Control for Walkway Locomotion Interface
}

\author{
Guillaume Millet \\ Sensorimotor Systems Laboratory \\ University of British Columbia \\ Vancouver, Canada \\ Email: gmillet@cs.ubc.ca
}

\author{
Martin J.-D. Otis \\ REPARTI Center, Department of Applied Science \\ University of Quebec at Chicoutimi \\ Saguenay, Canada \\ Email: martin.otis@uqac.ca
}

\author{
Jeremy R. Cooperstock \\ Centre for Intelligent Machines \\ McGill University \\ Montreal, Canada \\ Email: jer@cim.mcgill.ca
}

\begin{abstract}
Falls represent a major challenge to mobility for the elderly community, a point that has motivated various studies of balance failures. To support this work, we are interested in mechanisms for the synthesis of ground environments that can be controlled to exhibit dynamic friction characteristics. As a first step, we investigate the design and development of such a variable-friction device, a hybrid locomotion interface using a cable-driven vibrotactile mechanism. Measurements on our prototype, consisting of an aluminum tile covered with lowfriction polytetrafluoroethylene (PTFE), demonstrate that it can effectively simulate a low coefficient of static friction. As part of the design, we also investigated the role that induced vibration plays in modifying the coefficient of friction. Measurements of sliding on a PTFE-covered tile in a tilted configuration showed a significant influence of normal low-frequency vibration, particularly for frequencies around $20 \mathrm{~Hz}$, regardless of the user's weight.
\end{abstract}

Index Terms-Friction, vibrotactile, locomotion interface, walkway, cable-driven.

\section{INTRODUCTION}

Immersive virtual environments containing a multimodal floor interface with a high-fidelity vibrotactile floor have been developed recently to simulate virtual ground surfaces such as ice or snow [1]. The addition of variable friction to the floor surface would not only improve the realism of walking on natural ground surfaces but also facilitate the interaction with virtual objects requiring a sliding motion such as sliders, touch screen displays, or other foot-based control interfaces [2].

Variable-friction floor devices (VFFD) have great potential in other virtual-reality applications. For example, in gait or balance rehabilitation therapy, the use of a VFFD could provide a cost-effective way to simulate surfaces with different degrees of slipperiness in order to aid and evaluate strength development of the motor movements related to postural stability [3].

VFFDs also have potential to provide insight for biomechanical studies related to slips and falls. Indeed, one of the most hazardous conditions is melting ice where the very top layer is water [4]. Human-centered approaches have been used to estimate slipping and falling hazards and risks [5], to evaluate shoe characteristics and users' perception of stability [6], and to analyze biomechanics [7] and motor patterns [8] when anticipating or walking on slippery floors. One of the challenges of such biomechanical studies, however, is reproducing the unexpected nature of real-life slipping accidents
[7], a problem that can be solved by VFFDs presented in this paper.

Designing a floor device able to generate a wide range of friction coefficients when people walk on it poses two interdependent issues. First, how to achieve very low friction at the lowest desirable levels of $\mu_{\mathrm{s}}$, and second, how to be able to vary this friction continuously in a controllable manner. Following the literature review, we describe our investigation into a low-friction display that retains vibrotactile capability, using low-friction coverings. As the device is planned to be combined with a kinesthetic platform able to display lowfrequency $(<40 \mathrm{~Hz})$ vibrations, the second part of the paper presents preliminary results on the influence of the user's weight and the amplitude and frequency of low-frequency vibrations normal to the surface on the coefficient of friction of the device.

\section{A. Related Work}

Friction is quantified with Coulomb's model in which a relative motion between two solids starts when the ratio of frictional (tangential) to normal forces exceeds a certain value called the coefficient of static friction $\left(\mu_{\mathrm{s}}\right)$. In the context of walking, friction is the force resisting the relative motion between the floor surface and a walker's shoe. For instance, rubber on dry asphalt has a $\mu_{\mathrm{s}}$ above 0.5 and rubber on melting ice at $-1^{\circ} \mathrm{C}$ drops the $\mu_{\mathrm{s}}$ to around 0.02 [9]. Although such low $\mu_{\mathrm{s}}$ surfaces would generally be considered a safety issue, some applications, for instance when studying slip-induced falls, ave this as the goal. With the exception of ice, most normal shoefloor interactions are not slippery, exhibiting a $\mu_{\mathrm{s}}$ above 0.3 .

Most experimental devices used in slips-related studies varied the slipperiness by changing the floor-footwearcontaminant conditions [10], [7], [11]: subjects walked along a walkway with different flooring material, e.g., vinyl, carpet, plywood, or concrete, over which a contaminant such as water, sand, or oil could be poured, and in different shoe conditions, e.g., different sole material, sole hardness, and heel height. Some researchers have instead used a roller-based apparatus [12]. Millet et al. proposed a mechanism to continuously modulate the friction using such a roller-based system. Although this can produce both high coefficients of friction when the rollers are locked and very slippery conditions when unlocked, 
it adds significant perceptual biases such as an uneven surface, vibrotactile noise, visibility and thus awareness of the danger, and friction anisotropy. A similar approach is to use a mobile platform atop a set of lockable low-friction linear bearings [13], which avoids some of the aforementioned drawbacks such as irregularity and visibility.

For the related problem of rendering virtual textures under the fingertip, variable-friction tactile displays have been used recently. Most of these devices use ultrasonic vibrations to vary the apparent coefficient of friction. According to Windfield et al. [14], this friction reduction seems to be caused by the creation of a compressed film of air between the vibrating surface and the finger touching it. Because the normal forces and sizes of feet are at least two orders of magnitude higher than those of fingers, this technique of friction reduction is not likely to scale to floor applications.

\section{B. Geometry of the locomotion interface}

A locomotion interface should include two platforms, one for each foot, fully constrained in six degrees of freedom. Other designs of locomotion interfaces using fully constrained platforms, such as the K-Walker [15] and the HapticWalker [16], use two similar platforms although the biomechanics of walking generate different foot trajectories that are different for each user [17].

A fully constrained walking platform is complex, requires significant installation space, and has high fabrication cost since the hardware must at least match the speed of human motion and support human weight. Finally, it requires an additional actuator for generating vibration under the foot, as this capability is not typically provided by conventional robotic hardware. Indeed, vibration often tends to damage the equipment. In order to overcome these issues, we propose the use of a hybrid geometry: 1) an under-constrained cable-driven platform for each foot as shown in Fig. 1 and 2) a tiltable tile in which the human weight is supported as shown in Fig. 2. The tile consists of four main components: a) the linear actuators that adjust the angle, b) an elastomer that enables vibration, c) an axis of rotation between each tile, and d) a vibrotactile actuator that generates the physical properties of different types of soil.

To enable interaction with virtual objects and adequately simulate different soil types, it is suggested that the platform under the foot is driven by four cables. The tensile force and the length of the cable together allow rendering physical properties of contact between foot and virtual object in three degrees of freedom (one rotation and planar motion). The cable is driven by a motorized reel.

For safety reasons, a tether is connected to the subject's hip. This tether, driven by four cables, is used to reduce the risk of falling and also for the washout algorithm, which simulates an infinite environment in a physical limited space, similar to the motorcycle simulator presented in reference [18]. The washout algorithm was previously used in another cable-driven haptic mechanism [19].

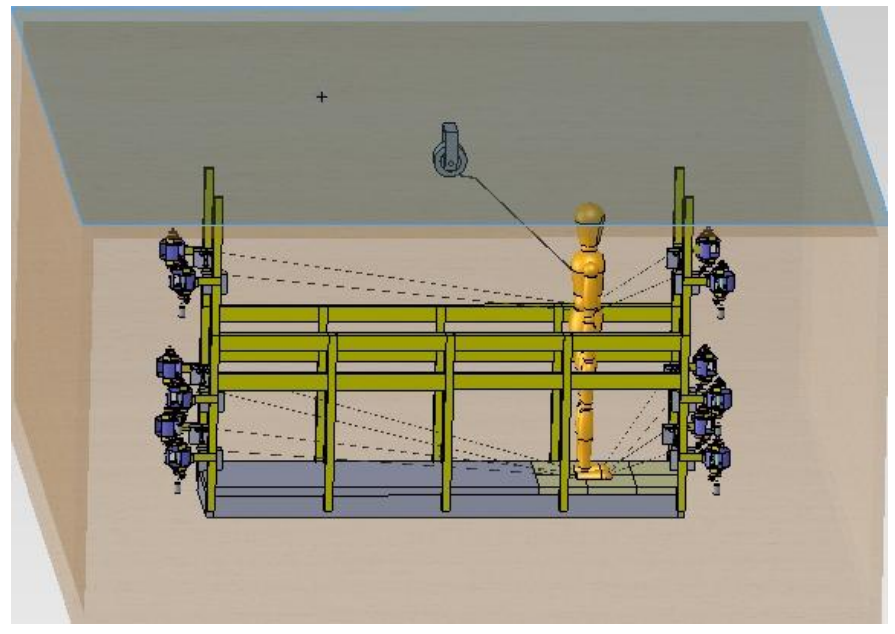

Fig. 1. Locomotion Interface Prototype

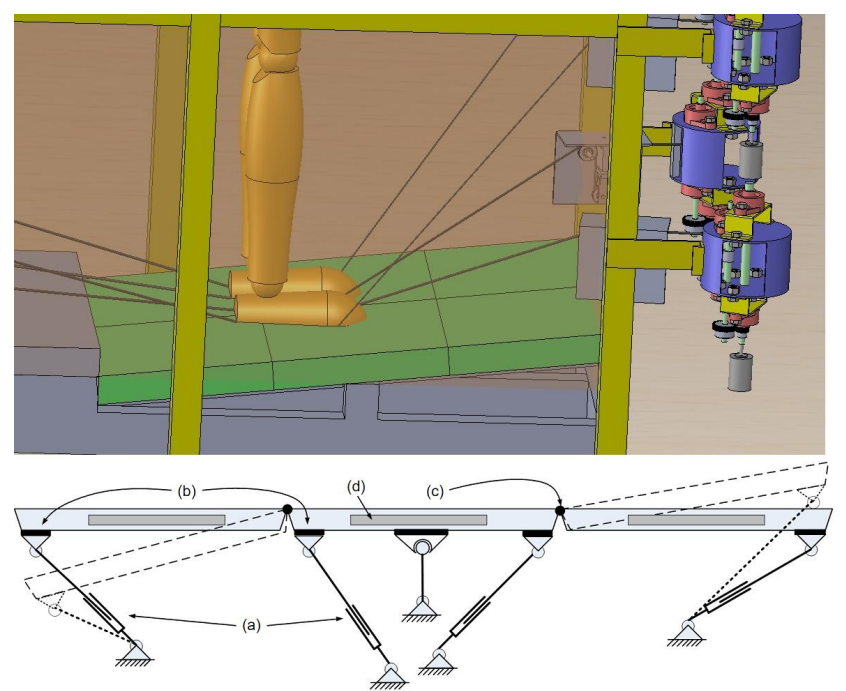

Fig. 2. Tilting mechanism to measure friction angles on the locomotion interface

In order to reduce the cost and size of motors, the subject's gait stance is assumed to be permanently in contact with some aluminum tiles. Each tile contains an actuator for rendering vibrotactile information related to the simulated ground surface. Simulating the haptic perception of ground movement, e.g., that of sand under the foot, as well as apparent characteristics of friction, is achieved by a mechanism with linear actuators as shown in Fig. 2.

\section{2D VARIABLE-FRICTION FLOOR DEVICE}

In mechanical systems design, reducing friction between surfaces is usually accomplished by one of the three following methods. First, one can place a lubricant such as oil, water, or grease between the two surfaces. This method is not considered here because of the impracticality of constantly renewing the lubrication as well as the difficulty in varying the friction of such a preparation. Another common method is to change the contact surfaces to naturally low-friction materials, such as 


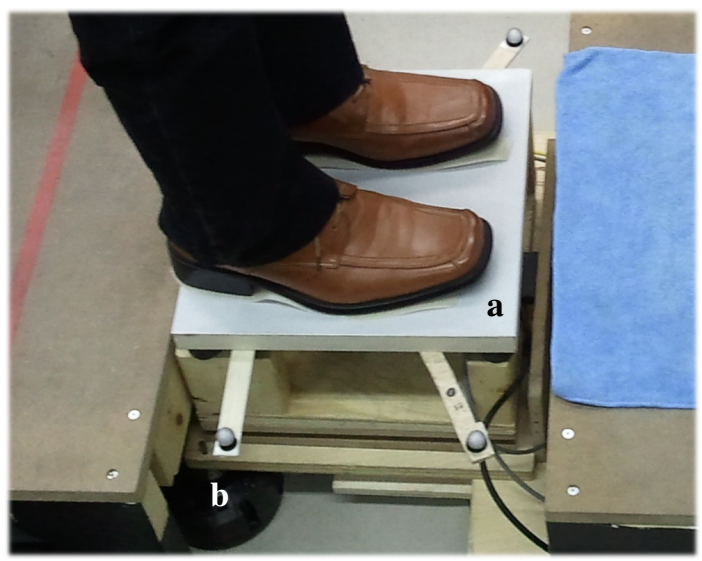

Fig. 3. Prototype of a PTFE-covered vibrotactile tile, made from the tiles described in reference [21] and fixed on a tilting mechanism to measure friction angles.

ultra-high-molecular-weight polyethylene (UHMWPE) used in synthetic ice rinks or polytetrafluoroethylene (PTFE) whose self-contact $\mu_{\mathrm{s}}$ ranges from 0.05 to 0.1 at low sliding speeds $(<0.1 \mathrm{~m} / \mathrm{s})$ [20]. The third way is to change sliding friction into rolling friction, which naturally has a lower resistance. Using devices such as rolling-element bearings often allows a $\mu_{\mathrm{s}}$ below 0.02 .

\section{A. Low-Friction Surface Coverings}

This design is based on a flat surface, which offers the advantage of being easily integrated into a virtual environment. The minimum friction achievable in this configuration is determined by the coefficient of friction of the material used to cover both the shoes and the tile.

To verify this, we constructed a prototype of slippery tiles covered with PTFE (see Fig. 3). Since the tiles are intended to be part of a vibrotactile display, the prototypes were built upon the vibrotactile tiles manufactured previously by our group [21]. For testing, the shoes were covered with PTFE sheets. Since the accumulation of dust between the surfaces has the unintended consequence of increasing the resistance of motion, the coverings need to be wiped clean regularly.

\section{B. Measurements of $\mu_{\mathrm{s}}$}

The coefficients of static friction $\mu_{\mathrm{s}}$ were calculated from empirical measurements of the friction angle $\varphi_{\mathrm{s}}$ at which one shoe starts sliding, such as $\mu_{\mathrm{s}}=\tan \varphi_{\mathrm{s}}$. Figure 3 illustrates the apparatus, which was mounted at an initial tilt angle of $4^{\circ}$. The tile a was tilted by a linear actuator b (D-Box Odyssey) which was able to tilt the prototype an additional $3.2^{\circ}$, thus capable of achieving a maximum total angle of $7.2^{\circ}$. Tilting was performed with a slow ramp at a speed of $0.2^{\circ} / \mathrm{s}$. The orientation of the tile and the movement of shoes were measured by an OptiTrack motion capture system.

Five male subjects weighing between 50 and $80 \mathrm{~kg}$ took part in the experiment. Each participant was given a pair of hard-soled men's dress shoes in his size and covered with the appropriate PTFE sheets. Participants were asked to stand on

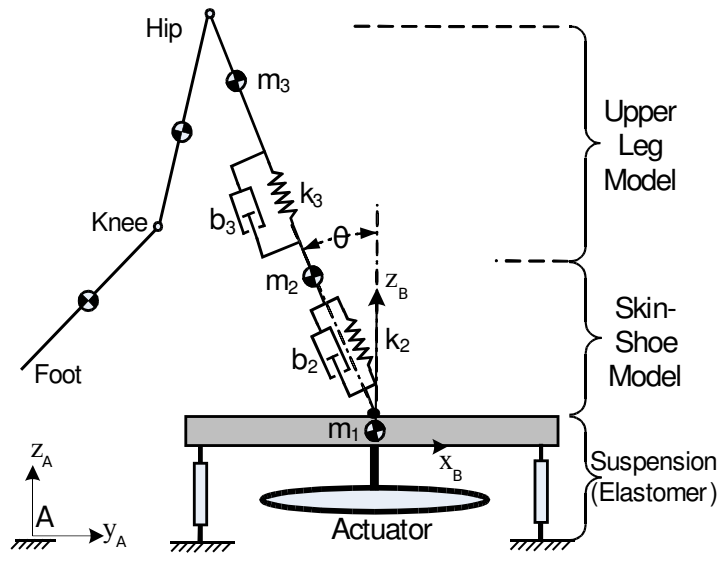

Fig. 4. A simplified rheological model of the user coupled to the friction display device.

the tile and to maintain their vertical posture during the tilting of the tile.

A coefficient of $0.11 \pm 0.01$ was measured. This value is on the higher end of common measured value for this material. However, we suspect that reducing the contact area, hence increasing contact pressure would result in lower coefficients of friction, closer to that reported by Dupont of around 0.05 [20].

\section{INFLUENCE OF LOW-FREQUENCY VIBRATIONS ON FLOOR FRICTION}

Influence of floor vibrations on friction has received some attention in the field of civil engineering for the development of friction-based isolators involved in the structural safety and reliability of buildings in regions susceptible to earthquakes [22], [23]. It may be desirable to quantify the variations in the friction display response due to vibrations, and to compensate for them if they significantly affect the desired level of friction. However, we are not aware of any prior studies of this nature.

The contact dynamics between the shoes and floor varies with the compliance of the shoe and lower limb, in a way that certain frequencies and amplitudes of floor vibration can significantly decrease the normal forces at the contact, and in turn, simulate a lower coefficient of friction. Figure 4 shows a simple rheological model of the lower limb made of secondorder models of the skin and of the leg [24]. Physiologically, biomechanical properties such as leg joint stiffness cannot be regarded as static or linear during movement, as muscular activation regularly modulates the dynamic properties of the lower limbs during activities on foot [25], [26], [12]. In particular when people walk on a slippery floor, they adopt a gait mode that tends to keep the center-of-body mass centered over the supporting limbs and to increase limb stiffness [8]. Their postures stay close to the standing posture. Therefore, we focused our preliminary experiments on the variation of shoe-floor friction with standing people. 


\section{A. Experimental setup and procedure}

The linear actuators used in the platform described above enables application of normal low-frequency vibrations up to $100 \mathrm{~Hz}$. The command signal was provided as an audio signal to the controller of the actuators (model KAI-4P). In order to measure the normal acceleration of the tile under the shoes, a single accelerometer was insufficient due to the presence of angular acceleration. First, the low-frequency vibration actuators apply a force not at the center of the tile but on its side, which creates an angular acceleration. Secondly, the suspension elements under the tile, which are required for the vibrotactile display, permit angular vibrations with regard to the base, depending on the position of the feet. An angular acceleration of the tile means that normal acceleration differs according to the position on the tile. With some assumptions, the normal acceleration at each point of the tile can be calculated from the normal accelerations measured at three non-collinear points. Therefore, three accelerometers (Analog devices model ADXL 320 with a $1 \mathrm{kHz}$ bandwidth) were attached under the tile on three different sides.

Assuming that the square of angular velocities can be neglected, the acceleration $a_{\mathrm{z}}$ normal to the tile at position $(x, y)$ can be calculated from the normal accelerations $a_{i z}$ measured by the three accelerometers, by resolving the following linear system of three equations:

$$
a_{\mathrm{z}}+\dot{\omega}_{\mathrm{x}}\left(y-y_{i}\right)-\dot{\omega}_{\mathrm{y}}\left(x-x_{i}\right)=a_{i \mathrm{z}} \quad \text { for } \mathrm{i}=\{1,2,3\},
$$

where $\left(x_{i}, y_{i}\right)$ are the coordinates of accelerometer $i$, and $\dot{\omega}_{\mathrm{y}}$ and $\dot{\omega}_{\mathrm{y}}$ are the angular accelerations of the tile around its tangential axes. Assuming that the three accelerometers are not aligned, the solution of the system is

$$
\begin{aligned}
a_{\mathrm{z}} & =\left(a_{1 z}\left(x\left(y_{2}-y_{3}\right)-y\left(x_{2}-x_{3}\right)+x_{2} y_{3}-y_{2} x_{3}\right)\right. \\
& +a_{2 z}\left(x\left(y_{3}-y_{1}\right)-y\left(x_{3}-x_{1}\right)+x_{3} y_{1}-y_{3} x_{1}\right) \\
& \left.+a_{3 z}\left(x\left(y_{1}-y_{2}\right)-y\left(x_{1}-x_{2}\right)+x_{1} y_{2}-y_{1} x_{1}\right)\right) \\
& \frac{1}{x_{1}\left(y_{2}-y_{3}\right)+x_{2}\left(-y_{1}+y_{3}\right)+x_{3}\left(y_{1}-y_{2}\right)}
\end{aligned}
$$

The acceleration of the tile was computed at the center of pressure of the forces applied by the shoes on the tile. Force sensing was performed via four load cells (Measurement Specialties model FX19) located under each corner of the support of the tile. Analog data from the force sensors were amplified and digitized via an acquisition board (National Instruments model USB-6218).

Five males participated in the experiment, the same group involved in the measurements of $\mu_{\mathrm{s}}$. All gave their informed consent in agreement with university ethics guidelines. They were asked to stand in the middle of the tile with the PTFEcovered shoes and to maintain their standing posture while sliding. As illustrated in Figure 5, safety bars were available on both sides of the platform in case the participants lost their balance. After each test, they were asked to return to the initial position for the following test.

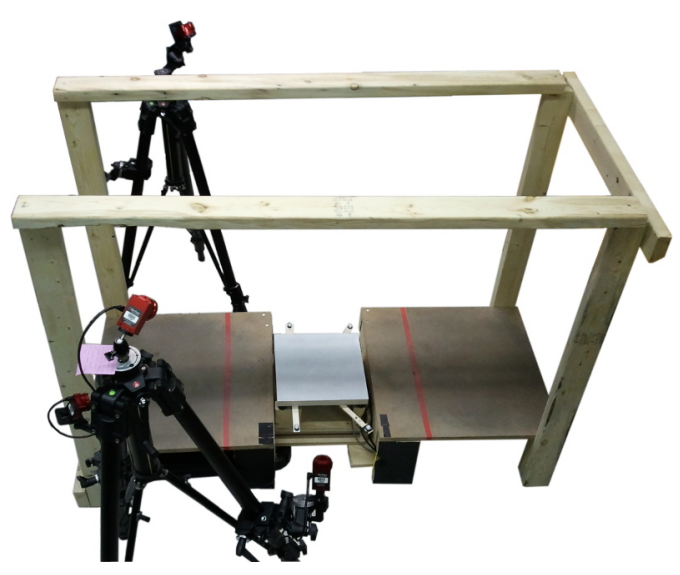

Fig. 5. Apparatus for producing low-frequency vibrations

\section{B. Influence of vibration frequency}

Seven patterns of sinusoidal vibrations lasting five seconds with frequencies ranging from 5 to $35 \mathrm{~Hz}$ were tested. The tile was tilted by $5^{\circ}$, which is close to the friction angle measured previously. The vibration intensity was the maximum allowed by the controller. The acceleration measured at the center of pressure $(\mathrm{CoP})$ was approximately $0.8 \mathrm{~g} \pm 0.1 \mathrm{~g}$, with the lowest accelerations when the $\mathrm{CoP}$ was close to the non-actuated side.

Figure 6 shows the displacement of the position of the CoP in response to different vibration frequencies for one subject. A dashed line indicates the average velocity of the displacement. The analyzed displacement was terminated at $10 \mathrm{~cm}$. The result of this analysis for the five subjects is given in Figure 7. In general, the subjects slid faster when the frequency of vibration was $20 \mathrm{~Hz}$. The greater $\mathrm{CoP}$ velocities at $5 \mathrm{~Hz}$ are likely due to the large amplitude of displacement that tilted the tile above the friction angle.

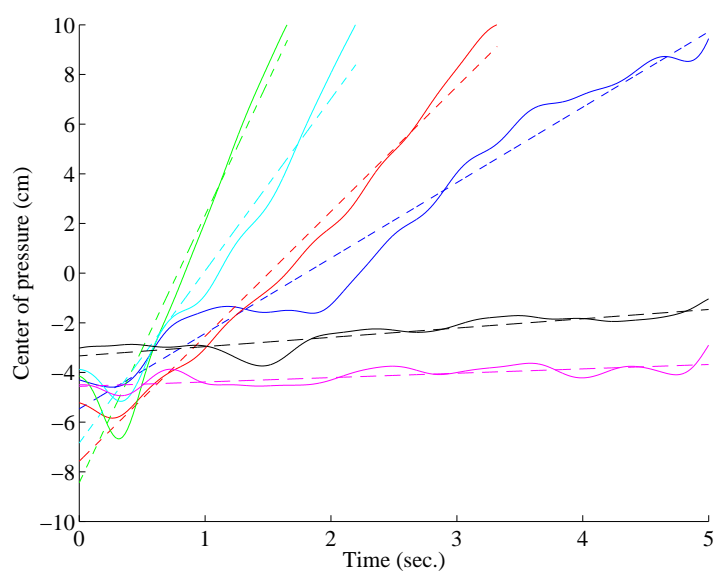

Fig. 6. Measurements of position of the center of pressure with different vibration frequencies from 5 to $35 \mathrm{~Hz}$.

\section{CONClusions ANd Future wORK}

We proposed and discussed the design of a locomotion interface with a variable-friction floor device using low-friction 


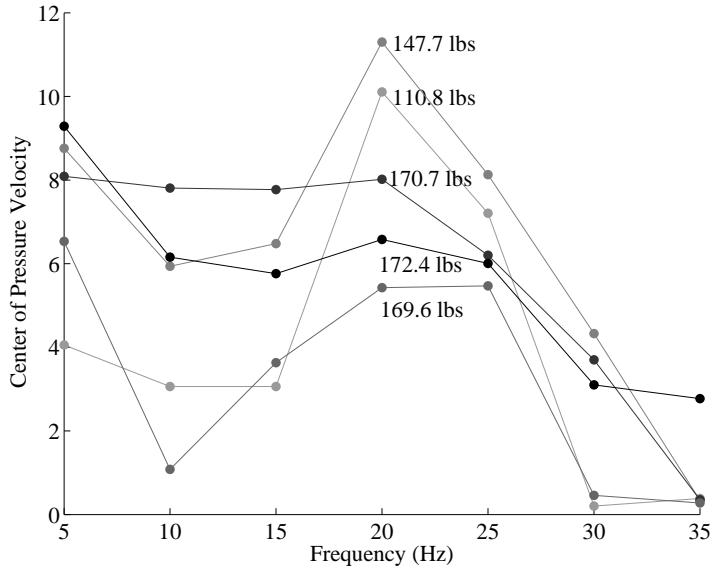

Fig. 7. Velocity of motion of the center of pressure of the participants.

coverings. As part of the design, we investigated the role that induced vibration plays in modifying the coefficient of friction. Measurements of sliding on a tile in a tilted configuration showed a significant influence of normal low-frequency vibration particularly for frequencies around $20 \mathrm{~Hz}$ whatever the user's weight.

Future work includes designing a variable-friction floor device by combining low- and high-friction surfaces in a controllable manner and investigating the influence of normal and tangential vibrations on shoe-floor friction with the different body postures involved in walking.

\section{ACKNOWLEDGMENT}

This work was supported by a grant from the Ministère du Développement économique, de l'Innovation et de l'Exportation in coordination with the European Commission Seventh Framework FET-OPEN project NIW: Natural Interactive Walking and by a post-graduate scholarship from the Fonds québécois de la recherche sur la nature et les technologies. The authors would like to thank Yon Visell for his foundational contributions to these efforts.

\section{REFERENCES}

[1] Y. Visell, A. Law, J. Ip, S. Smith, and J. R. Cooperstock, "Interaction capture in immersive virtual environments via an intelligent floor surface," in IEEE Virtual Reality (VR), Waltham, MA, USA, mar 2010, pp. 313-314.

[2] Y. Visell, S. Smith, A. Law, R. Rajalingham, and J. R. Cooperstock, "Contact sensing and interaction techniques for a distributed, multimodal floor display," in IEEE 3D User Interfaces (3DUI), Waltham, MA, USA, mar 2010, pp. 75-78.

[3] A. Mirelman, P. Bonato, and J. Deutsch, "Effects of training with a robot-virtual reality system compared with a robot alone on the gait of individuals after stroke," Stroke, vol. 40, no. 1, pp. 169-174, 2009.

[4] C. Gao and J. Abeysekera, "A systems perspective of slip and fall accidents on icy and snowy surfaces." Ergonomics, vol. 47, no. 5, pp. 573-598, Apr 2004.

[5] R. Grönqvist, J. Abeysekera, G. Gard, S. M. Hsiang, T. B. Leamon, D. J. Newman, K. Gielo-Perczak, T. E. Lockhart, and C. Y. Pai, "Humancentred approaches in slipperiness measurement." Ergonomics, vol. 44, no. 13, pp. 1167-1199, Oct 2001.
[6] J. C. Menant, S. D. Perry, J. R. Steele, H. B. Menz, B. J. Munro, and S. R. Lord, "Effects of shoe characteristics on dynamic stability when walking on even and uneven surfaces in young and older people," Archives of Physical Medicine and Rehabilitation, vol. 89, no. 10, pp. 1970-1976, 2008.

[7] R. Cham and M. S. Redfern, "Changes in gait anticipating slippery floors," Gait \& Posture, vol. 15, no. 2, pp. 159-171, 2002.

[8] G. Cappellini, Y. P. Ivanenko, N. Dominici, R. E. Poppele, and F. Lacquaniti, "Motor patterns during walking on a slippery walkway," Journal of Neurophysiology, vol. 103, no. 2, pp. 746-760, 2010.

[9] A. Roberts and J. Richardson, "Interface study of rubber-ice friction," Wear, vol. 67, no. 1, pp. 55-69, 1981.

[10] M. Llewellyn and V. Nevola, "Strategies for walking on low-friction surfaces," in International Conference on Environmental Ergonomics, 1992, pp. 156-157.

[11] D. T.-P. Fong, Y. Hong, and J.-X. Li, "Human walks carefully when the ground dynamic coefficient of friction drops below 0.41," Safety Science, vol. 47, no. 10, pp. 1429-1433, 2009.

[12] D. S. Marigold and A. E. Patla, "Strategies for dynamic stability during locomotion on a slippery surface: Effects of prior experience and knowledge," Journal of Neurophysiology, vol. 88, no. 1, pp. 339-353, 2002

[13] Y.-C. Pai, "Induced limb collapse in a sudden slip during termination of sit-to-stand," Journal of Biomechanics, vol. 32, no. 12, pp. 1377-1382, 1999.

[14] L. Winfield, J. Glassmire, J. E. Colgate, and M. Peshkin, "T-pad: Tactile pattern display through variable friction reduction," in Second Joint EuroHaptics Conference and Symposium on Haptic Interfaces for Virtual Environment and Teleoperator Systems. World Haptics 2007, Mar. 2007, pp. 421-426.

[15] J. Yoon and J. Ryu, "A novel locomotion interface with two 6-dof parallel manipulators that allows human walking on various virtual terrains," International Journal of Robotics Research, vol. 25, no. 7, pp. $689-$ 708, 2006.

[16] S. Hesse, H. Schmidt, and C. Werner, "Machines to support motor rehabilitation after stroke: 10 years of experience in berlin," Journal of Rehabilitation Research and Development, vol. 43, no. 5, pp. 671 678, 2006.

[17] M. J.-D. Otis, S. Comtois, D. Laurendeau, and C. Gosselin, "Human safety algorithms for a parallel cable-driven haptic interface," in Brain, Body and Machine, ser. Advances in Intelligent and Soft Computing. Springer Berlin Heidelberg, 2010, vol. 83, pp. 187-200.

[18] C. Avizzano, F. Barbagli, and M. Bergamasco, "Washout filter design for a motorcycle simulator," in IEEE International Conference on Systems, Man, and Cybernetics, vol. 2, 2000, pp. 995-1000.

[19] M. J. Otis, V. Duchaine, G. Billette, S. Perreault, C. Gosselin, and D. Laurendeau, "Cartesian control of a cable-driven haptic mechanism," Advances in Haptics, InTech, pp. 75-102, 2010.

[20] DuPont Fluoroproducts, "Teflon PTFE properties handbook," DuPont, Tech. Rep. H-37051-3, 1996.

[21] Y. Visell and J. R. Cooperstock, "Design of a vibrotactile display via a rigid surface," in IEEE Haptics Symposium, Waltham, MA, USA, mar 2010, pp. 133-140.

[22] A. Pirrotta and R. A. Ibrahim, "Experimental investigation of frictionbase isolation," Probabilistic Engineering Mechanics, vol. 12, no. 3, pp. 125-136, 1997.

[23] A. G. Hernried and K.-M. Lei, "Semi-analytical techniques for the determination of the dynamic response of equipment in structures supported on coulomb friction elements," Earthquake Engineering \& Structural Dynamics, vol. 24, no. 6, pp. 801-810, 1995.

[24] M. G. Pandy and N. Berme, "Synthesis of human walking: A planar model for single support," Journal of Biomechanics, vol. 21, no. 12, pp. 1053 - 1060, 1988.

[25] D. P. Ferris, M. Louie, and C. T. Farley, "Running in the real world: adjusting leg stiffness for different surfaces," Proc Biol Sci., vol. 265, no. 1400, pp. 989-994, Jun. 1998.

[26] S. Rapoport, J. Mizrahi, E. Kimmel, O. Verbitsky, and E. Isakov, "Constant and variable stiffness and damping of the leg joints in human hopping," Journal of Biomechanical Engineering, vol. 125, pp. 507-514, 2001. 